\title{
Development of Simple Portable Blood Glucose Measuring Devices
}

\author{
Ying-Fu Su, ${ }^{1 \dagger}$ Shih-Hung Lin, ${ }^{2 \dagger}$ Sin-Liang Ou, ${ }^{3}$ and Yao-Chin Wang ${ }^{4 *}$ \\ ${ }^{1}$ Department of Emergency Medicine, Kuang Tien General Hospital, \\ 117 Shatian Rd., Shalu District, Taichung City 43303, Taiwan \\ ${ }^{2}$ Department of Electronics Engineering, National Yunlin University of Science and Technology, \\ 123 University Road, Section 3, Douliou, Yunlin 64002, Taiwan \\ ${ }^{3}$ Department of Biomedical Engineering, Da Yeh University, \\ 168 University Rd., Dacun, Changhua 51506, Taiwan \\ ${ }^{4}$ Department of Computer Science and Information Engineering, Cheng Shiu University, \\ 840 Chengcing Rd., Niaosong District, Kaohsiung City 83347, Taiwan
}

(Received December 18, 2020; accepted March 26, 2021)

Keywords: Arduino-based system, biomedical sensing, glucose concentration

Probing biomedical materials with multiple wavelengths can not only obtain an in-depth understanding of the detected biomedical materials but also differentiate the materials. Biomedical materials have different optical properties (e.g., absorption and induction) for different wavelengths. Therefore, to achieve this purpose, we present in this study our experimental results on fabricating portable biomedical sensing devices. In this research phase, we assembled two types of system. One system used light sources with multiple wavelengths and photodetectors detecting these wavelengths, with which we preliminarily tested the absorbance of glucose solutions with different concentrations. The other was an Arduino-based glucose sensing system.

\section{Introduction}

Different strategies, e.g., electrical-based and optical-based measurements, have been proposed for detecting biomedical materials. Optical detection utilizes the optical properties (e.g., absorption) of detected biomedical materials. Biomedical material detection is of increasing importance and has a wide range of applications, e.g., pathological examination, industrial chemical detection, and personal physiological monitoring. ${ }^{(1,2)}$ For instance, as shown in Fig. 1(a), hemoglobin exhibits different optical absorption characteristics ${ }^{(3)}$ for the oxy and deoxy states. Compared with the deoxy-hemoglobin state (deoxy-hemoglobin, Hb), oxy-hemoglobin (hemoglobin bound to oxygen, $\mathrm{HbO}_{2}$ ) has stronger absorbance in the near-infrared regime (wavelengths longer than $\sim 800 \mathrm{~nm}$ ) but weaker absorbance between 600 and $800 \mathrm{~nm}$. Both show similar absorbances for wavelengths shorter than $\sim 600 \mathrm{~nm}$. Therefore, probing hemoglobin with multiple wavelengths can differentiate its state.

Glucose has stronger absorption than water in some near-infrared wavelength ranges, e.g., 900-1000 nm [Fig. 1(b)]; ${ }^{(4-6)}$ thus, these ranges can be used for sensing glucose. Conventional

Joint first authors: Ying-Fu Su and Shih-Hung Lin

*Corresponding author: e-mail: y.c.wang@ieee.org

https://doi.org/10.18494/SAM.2021.3228 


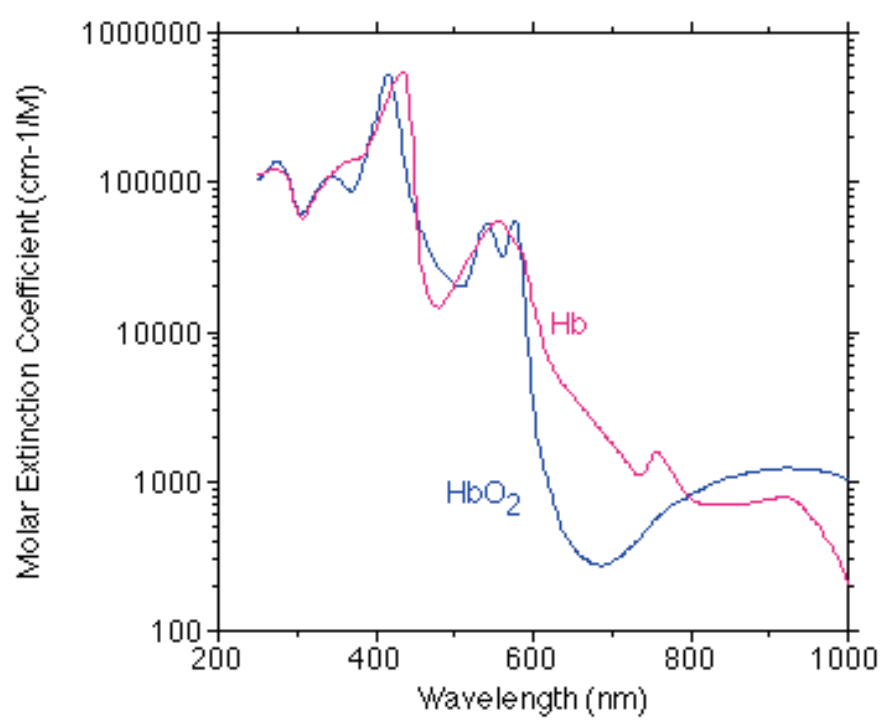

(a)

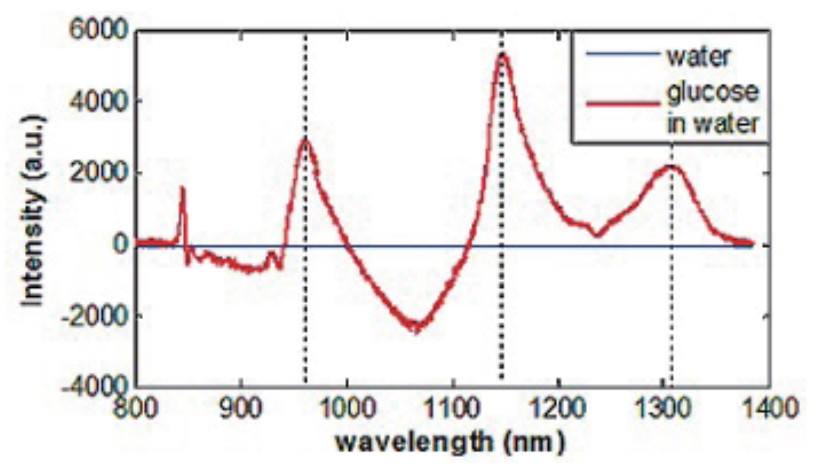

(b)

Fig. 1. (Color online) (a) Molar extinction coefficients of oxy-hemoglobin $\left(\mathrm{HbO}_{2}\right)$ and deoxy-hemoglobin $(\mathrm{Hb})$. $^{(3)}$ (b) Absorption spectrum of glucose. ${ }^{(4)}$

optical-based measurements typically use a single or single-band wavelength light source and a photodetector, which will limit the capability to differentiate biomedical materials. For example, both oxy-hemoglobin and deoxy-hemoglobin absorb light in the range of 900-1000 nm, although oxy-hemoglobin has stronger absorption. Hence, for a mixture of oxy-hemoglobin and deoxyhemoglobin, it is difficult to quantitatively extract the absorption of each component using a single or single-band wavelength. However, it is possible to resolve this issue by using multiple (band) wavelengths. In addition to detectability, portability is another consideration for modern biomedical sensing systems. Ideally, a sensing system can be integrated as a chip.

\section{Experiments}

In this study, we present our preliminary results of fabricating a portable multiple-wavelength biomedical sensing system. In this phase, we performed preliminary tests on glucose solutions with different concentrations. The results showed good linearity of the absorbance with the 
glucose concentration. We also measured the absorbance using a broadband white light source and a spectrometer, which also exhibited good linearity with the glucose concentration but had a different slope from that of the multiple-wavelength sensing system. The cause of the difference in slope is still under investigation but may be related to the difference in the optical design.

Figure 2(a) shows a schematic of the preliminary design of our multiple-wavelength biomedical sensing system. The light source, located at the top, is composed of a multiplewavelength LED array with emission colors of blue, green, red, and near infrared. Their emission spectra are plotted in Fig. 3(a). The bottom of the system comprises a detector array, where the photodetectors denoted as B, G, R, and IR have a response range and peak wavelength of 400-540 and $460 \mathrm{~nm}, 480-600$ and $540 \mathrm{~nm}, 590-720$ and $660 \mathrm{~nm}$, and 880-1050 and 940 $\mathrm{nm}$, respectively, which were obtained from the detector datasheets and are replotted in Fig. 3(b). Thus, the response ranges of the individual photodetectors basically match the emission spectra of the corresponding LEDs. Both the LED and photodetector arrays are controlled by an Arduino circuit board (model Arduino UNO Rev3). The LEDs are turned on for $0.5 \mathrm{~s}$ in the sequence of near infrared, red, green, and blue, and only the single corresponding photodetector is read while the LED is on. The sample, which is glucose solution in this study, is placed between the LED and photodetector arrays. The distance $d$ between the sample and photodetector is set to 7 or $4.2 \mathrm{~cm}$ to examine the effect of the optical design, as discussed later.

In addition, we fabricated another Arduino-based system, as shown in Fig. 2(b), to verify the performance of the multiple-wavelength biomedical sensing system. The multiple-wavelength LED array was replaced by a broadband light source (tungsten lamp) with emission light coupled into a fiber. After passing through the sample, the light was collected by a fiber through a collimated lens, then sent to an Ocean Optics spectrometer.

To preliminarily test the proposed multiple-wavelength biomedical sensing system, glucose solutions of different concentrations were used as samples, which were prepared by adding different weights of glucose to a fixed volume $(20 \mathrm{ml})$ of deionized (DI) water. The pure DI water was used as a reference sample. The optical density (OD) of the glucose solution was

(a)

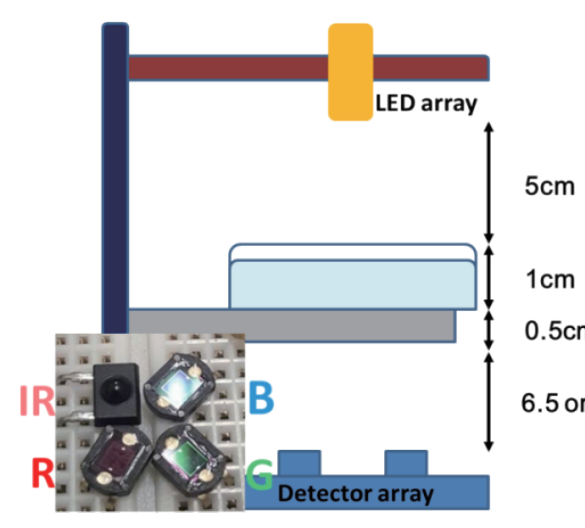

(b)

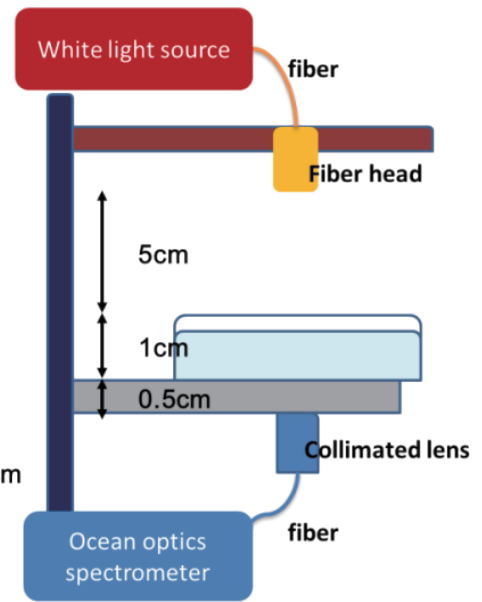

Fig. 2. (Color online) (a) Schematic of the preliminary design of our multiple-wavelength biomedical sensing system. (b) Another biomedical system with a white light source and a spectrometer. 

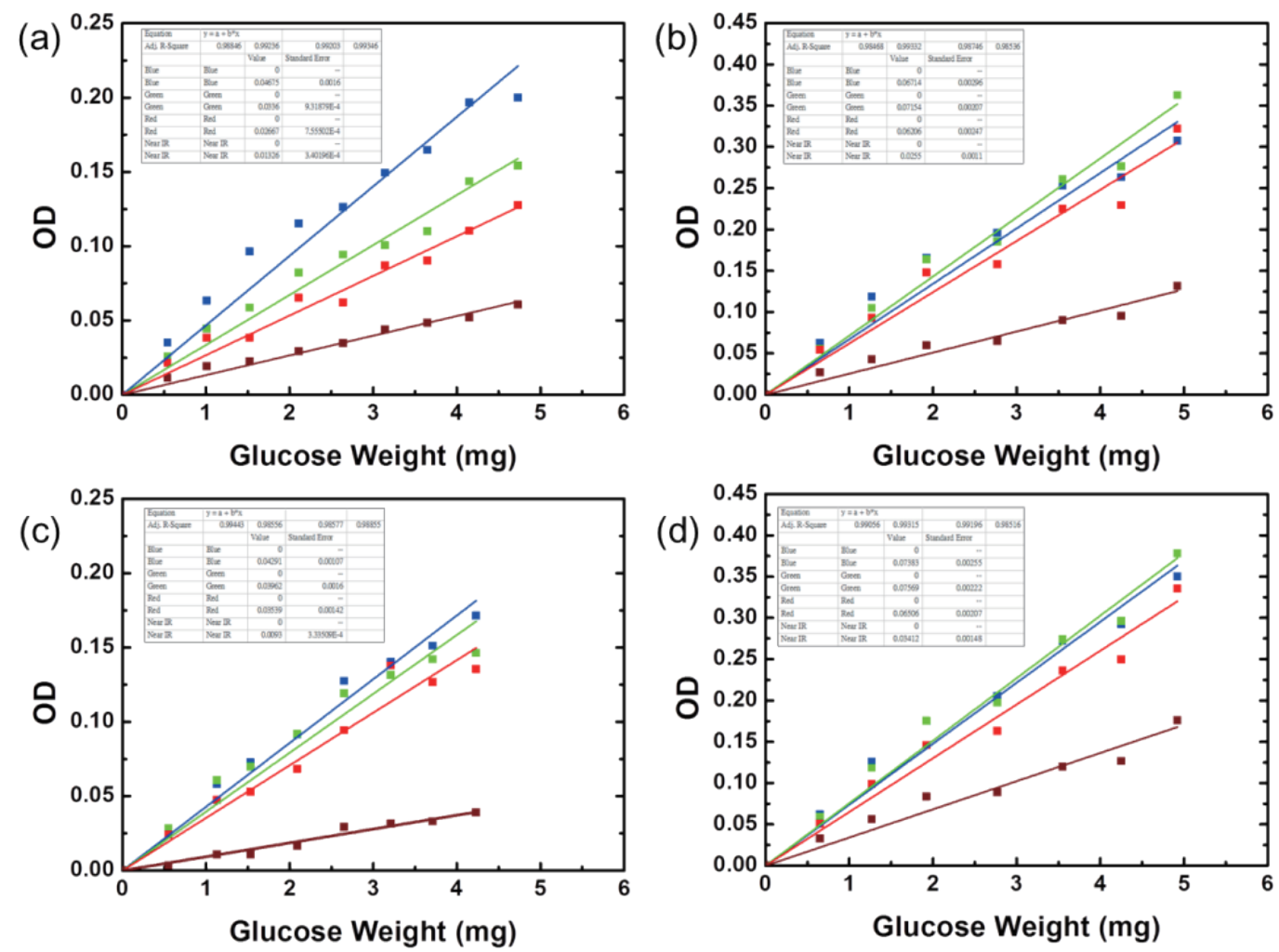

Fig. 3. (Color online) Optical density vs glucose weight for different colors (blue, green, red, near IR) measured by (a) multiple-wavelength sensing system with $d=7 \mathrm{~cm}$, (b) spectrometer system (single wavelength), (c) multiplewavelength sensing system with $d=4.2 \mathrm{~cm}$, and (d) spectrometer system (integrated spectral range).

calculated using $O D=\log I_{0}-\log I$, where $I_{0}$ and $I$ are the transmitted light intensities of the DI water and glucose solution, respectively.

\section{Results and Discussion}

Figure 3(a) shows the measured OD (for $d=7 \mathrm{~cm}$ ) of different glucose concentrations (different glucose weights) for blue, green, red, and near-infrared wavelengths. The OD for all wavelengths shows good linearity with the glucose concentration. The slope decreases from 0.0468 to 0.0133 as the wavelength increases from blue to near infrared. As shown in Fig. 3(b), the OD for different wavelengths $(438.2,566.24,688.32$, and $988.02 \mathrm{~nm})$ measured by an Ocean Optics spectrometer also exhibits good linearity with the glucose concentration. However, the slopes for the wavelengths of blue $(438.2 \mathrm{~nm})$, green $(566.24 \mathrm{~nm})$, and red $(688.32 \mathrm{~nm})$ are nearly identical and are larger than that for near infrared $(988.02 \mathrm{~nm})$. Rather than calculate the ODs using a single wavelength, the ODs were also calculated using the integrated spectral ranges of 415.23-520.43, 495.38-585.67, 615.4-705.65, and 900.2-1049.75 $\mathrm{nm}$ for the corresponding photodetectors of $B, G, R$, and IR. The results were similar to those obtained from a single wavelength. This phenomenon is considerably different from that observed using the proposed multiple-wavelength sensing system. 
To clarify the reason for this phenomenon, we decreased the distance $d$ from 7 to $4.2 \mathrm{~cm}$ and remeasured the OD of different glucose concentrations using the multiple-wavelength sensing system. As shown in Fig. 3(c), the OD still exhibited good linearity for the blue, green, red, and near-infrared wavelengths. However, the slopes for the blue, green, and red wavelengths were nearly the same and were larger than that for the near-infrared wavelength, which is consistent with the results obtained using the spectrometer system. In addition, the slope for near infrared was basically unchanged for the different values of $d$. This observation is associated with the effect of light scattering since the calculated OD includes absorption as well as light scattering. This argument is based on two points: (1) for $d=7 \mathrm{~cm}$, the slope decreases as the wavelength increases, which is consistent with the decrease in light scattering with increasing wavelength; ${ }^{(7)}$ (2) glucose has a certain amount of absorption in the near-infrared regime but no absorption in the spectral range between blue and red. However, for the blue, green, and red lights, the measured ODs were larger than that for the near-infrared light.

These results also indicate that light scattering can be used as another way to differentiate biomedical materials, but more investigation is still required. Although by shortening the distance $d$, the observation using the proposed multiple-wavelength sensing system was similar to the phenomenon observed using the spectrometer system, the slopes extracted using this sensing system for all wavelengths were still smaller than those obtained using the spectrum system. This difference in the slopes may be related to the difference in the optical design between the two systems, but more investigation is required to confirm this.

Figure 4(a) shows a schematic of the preliminary design of the second Arduino-based sensing system. The biomedical system shows the readout value from Arduino IDE for Windows in Fig. 4(b). Figure 5(a) shows boxplots of the glucose weight for different samples measured by the multiple-wavelength sensing system. Figure 5(b) shows the measured data for the Arduino-based system. Table 1 shows a comparison of the performances of the proposed system with previous systems. We also found that the scattering of light may affect the measurement and probably can serve as another way to differentiate biomedical materials. Note that by replacing the Arduino circuit board with a wireless function, the fabricated system can become a portable wireless sensing system. The size of the system can also be reduced further.

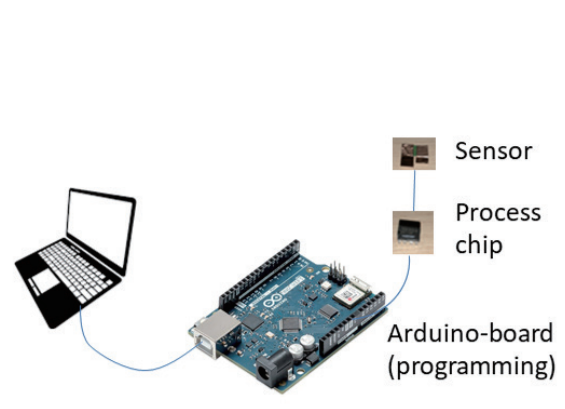

(a)

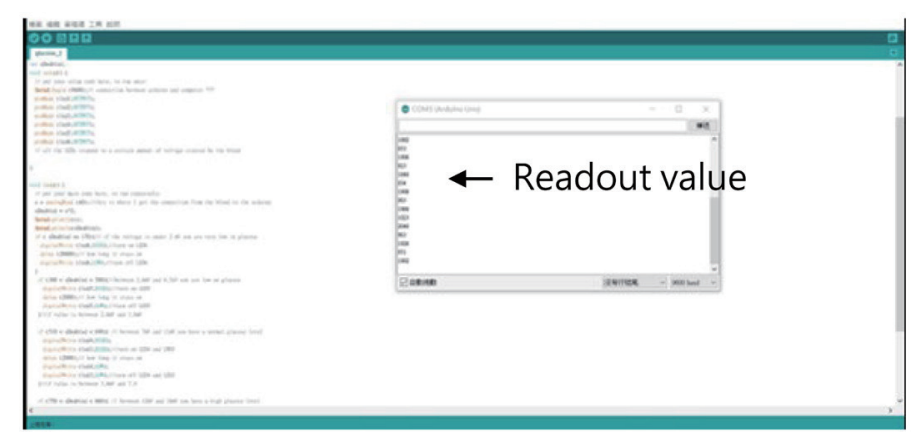

(b)

Fig. 4. (Color online) (a) Schematic of the preliminary design of the other biomedical sensing system. (b) Biomedical system with a readout value based on Arduino. 


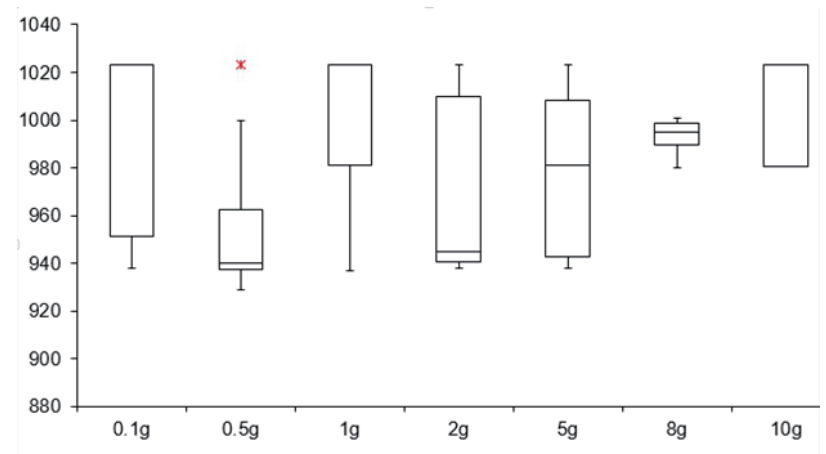

(a)

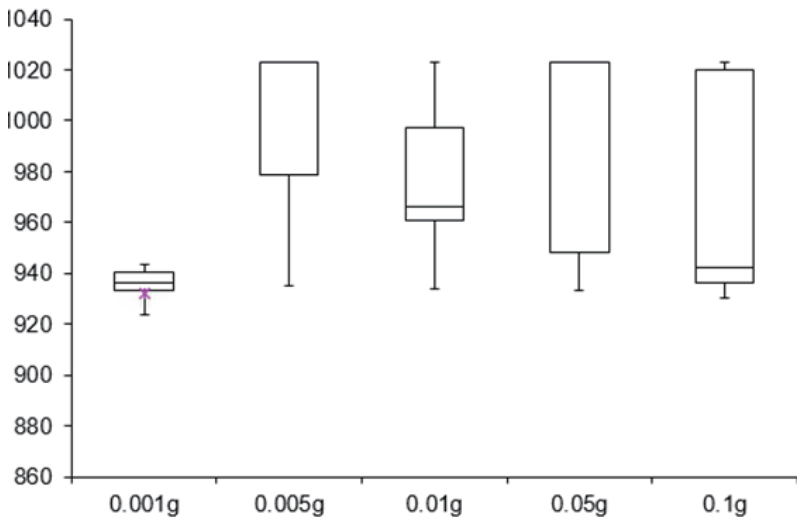

(b)

Fig. 5. (Color online) Boxplots of glucose weight for different samples measured by (a) multiple-wavelength sensing system and (b) Arduino system.

Table 1

Performance comparison of glucose sensing system. ${ }^{(8-10)}$

\begin{tabular}{lccc}
\hline Reference & Basic sensing mechanism & Response time (min) & Selectivity \\
\hline$(8)$ & Surface stress change & $\sim 39$ & Yes \\
$(9)$ & Volume change & $\sim 121$ & No \\
$(10)$ & Pressure change & $40-80$ & Yes \\
Proposed system & Optical/electrical change & $<1$ & Yes \\
\hline
\end{tabular}

\section{Conclusion}

We fabricated a portable multiple-wavelength biomedical sensing system by assembling LEDs of multiple colors and photodetectors to detect their wavelengths. To evaluate our preliminary system, we measured the OD of glucose solutions with different concentrations, which exhibited good linearity with the glucose concentration. In addition, we also measured the absorbance of the glucose solutions using a broadband white light source and a spectrometer. This results also exhibited linearity but with different slopes of the absorbance as a function of the glucose concentration, which confirmed the linearity obtained from the proposed sensing system. The difference in the slopes may be related to the difference in the optical design between these two systems. 


\section{Acknowledgments}

This work was supported by MOST of Taiwan under Contract No. MOST-1082637-E-230-002. It was also partially funded by industrial cooperation with Gadgletech under contract no. Yuntech 109-320 and Huo-Zhang Zong Research Center.

\section{References}

1 N. Reta, A. Michelmore, C. Saint, B. P. Simon, and N. H. Voelcker: Biosens. Bioelectron. 80 (2016) 47.

2 O. Syshchyk, V. A. Skryshevsky, O. O. Soldatkin, and A. P. Soldatkin: Biosens. Bioelectron. 66 (2015) 89.

3 Optical Absorption of Hemoglobin: http://omlc.org/spectra/hemoglobin/index.html (accessed December 2020).

4 Glucose Spectrum: http://www.andor.com/learning-academy/spectral-response-of-glucose-spectral-responsewithin-optical-window-of-tissue (accessed December 2020).

5 S. Kamasahayam, S. Haindavi, B. Kavala, and S. R. Chowdhury: 7th Int. Conf. Sensing Technology (2013) $627-631$.

6 R. J. McNichols and G. L. Cote: J. Biomed. Opt. 5 (2000) 5.

7 E. Hecht: Optics (Addison Wesley, 2001) 4th ed.

8 J. Pei, F. Tian, and T. Thundat: Anal. Chem. 76 (2004) 292.

9 T. Nagakura, K. Ishihara, T. Furukawa, K. Masuda, and T. Tsuda: Sens. Actuators, B 34 (1996) 229.

10 G. Lin, S. Chang, H. Hao, P. Tathireddy, M. Orthner, J. Magda, and F. Solzbacher: Sens. Actuators, B 144 (2010) 332. https://dx.doi.org/10.1016\%2Fj.snb.2009.07.054 